\title{
Big Data Tracking and Automatic Measurement Technology for Unmanned Aerial Vehicle Trajectory Based on MEMS Sensor
}

Jun Xing ( $\nabla$ xingjun@dlpu.edu.cn )

Dalian Polytechnic University

\section{Xinzhe Wang}

Dalian Polytechnic University

Jie Dong

Dalian Polytechnic University

\section{Research Article}

Keywords: MEMS sensor, Unmanned aerial vehicle (UAV) trajectory, Big data tracking, Automatic measurement

Posted Date: April 22nd, 2021

DOI: https://doi.org/10.21203/rs.3.rs-410175/v1

License: (c) (i) This work is licensed under a Creative Commons Attribution 4.0 International License. Read Full License

Version of Record: A version of this preprint was published at Soft Computing on March 23rd, 2022. See the published version at https://doi.org/10.1007/s00500-021-06457-y. 


\title{
Big data tracking and automatic measurement technology for unmanned aerial vehicle trajectory based on MEMS
}

\author{
sensor \\ Jun Xing*, Xinzhe Wang, Jie Dong \\ Institute of Information Science and Engineering, Dalian Polytechnic University, Dalian 116034, \\ China \\ Jun Xing*, E-mail: xingjun@dlpu.edu.cn \\ Xinzhe Wang, E-mail: wxzagm@dlpu.edu.cn \\ Jie Dong, E-mail: dongjie@qq.com
}

\begin{abstract}
Due to the high cost and large error of traditional UAV big data tracking and automatic measurement technology, a method of big data tracking and automatic measurement for UAV trajectory based on MEMS sensor was put forward. The iterative learning control algorithm was used to estimate the repetitive disturbance and modeling error of system based on the simplified dynamics model of four-rotor helicopter and the optimal estimation characteristics of Kalman filter. The discrete equation of quadratic performance function in time domain was selected to compensate the estimated model error disturbance, and then the big data tracking was completed. Based on the data of gyroscope, the quaternion differential equation was established. The differential equation was solved by first-order Picard method, and a set of quaternion data was obtained. The gradient descent method was used to process the acceleration data and magnetic data, and thus to get the optimal quaternion. Finally, the measurement results were obtained by fusing the two quaternions with MEMS sensors. Simulation results prove that the proposed method can obtain the trajectory tracking data and measurement information of UAV accurately.
\end{abstract}

Key words: MEMS sensor; Unmanned aerial vehicle (UAV) trajectory; Big data tracking; Automatic measurement

\section{Introduction}

The quad-rotor helicopter has a long history. It was originated in 1907. In the early stage, the lack of mature technology lead to slow development. In recent years, the progress of technology of aerial robot gradually brings huge application value and broad development prospects to quad-rotor helicopter (Zuo et al. 2019; He et al. 2016; Lei et al. 2011). Because the quad-rotor helicopter is a non-linear system and each channel has strong coupling characteristic, the design of flight control system is complex. In addition, quad-rotor aircraft tends to be miniaturized gradually. Due to small volume and low power consumption, MEMS sensor is applied in controller, but the measured values of sensor have large deviation. Therefore, it is particularly important to analyze the sensors (Peng et al. 2019; Niu et al. 2019), calibrate and integrate the sensor data.

In recent years, relevant scholars have made some significant research results. For example, Dai Shuxian put forward a target tracking and positioning algorithm based on RPCCA (Dai et al. 2019; Jiang et al. 2015). The regularization was used to analyze the constraint component and achieve the accurate target recognition of UAV. WiFi was adopted for stable and real-time transmission of big data. It can complement the satellite-based positioning algorithm. On this basis, the spread spectrum communication was adopted for accurate transmission of important data, and thus to improve the anti-interference performance of system.A tracking control method of UAV 
heterogeneous time-varying formation for air ground cooperative operation proposed by Zhou Siquan et al.The single kinematics and dynamics modeling of UAV is carried out. The whole movement track of UAV formation is described by virtual leader. Based on the distributed observer of virtual leader state, the UAV distributed formation tracking controller is constructed, and the selection method of each parameter in the formation tracking controller is obtained (Zhou et al. 2019). Nie Guanghua put forward a method of tracking the landing trajectory of UAV (Nie 2019). This method analyzed the landing process and requirement of UAV, simulated the landing trajectory of quad-rotor UAV, established the landing motion model of quad-rotor UAV by nonlinear equation including Gaussian noise, determined the landing measurement parameters, and determined the direction angle of reference trajectory motion. The tracking error of UAV landing trajectory was corrected in real time. Finally, the tracking result was obtained. The above methods can't meet the development needs at this stage. Therefore, the UAV trajectory big data tracking and automatic measurement method based on MEMS sensor was presented. Simulation results prove the effectiveness and superiority of the proposed method.

\section{Method}

\subsection{Big data tracking of UAV trajectory}

The kinetic model of quad-rotor unmanned helicopter is the basis and premise of the research on flight control algorithm. The correctness of model is very important to the design of control system. Firstly, the basic working principle is introduced, and the coordinate transformation matrix of airframe coordinate system and inertial coordinate system is defined (Pang et al. 2018). In addition, the mathematical model of system is established by the knowledge of rigid mechanics and Newton's laws (Zhai et al. 2014).

The quad-rotor unmanned helicopter is a system that four propellers are fixed at the end of an "X" or "cruciform" rigid structure. The propellers of flight system are symmetrically distributed at the front, rear, left and right ends on airframe, and the four rotors are on the same plane. The power of flight comes from four motors rotating at high speed. The attitude and trajectory of quad-rotor air vehicle can be controlled by adjusting the rotational speed of propellers. The rotor 2 and rotor 4 rotate counterclockwise, while rotor 1 and rotor 3 rotate clockwise. The pitching motion and forward motion can be formed by changing the speed of propeller 1 and propeller 3. Similarly, the rolling motion and lateral motion can be generated by changing the speed of propeller 2 and propeller 4 . Thus, the lift and attitude control force of aircraft can be formed.

Because the quad-rotor helicopter has six degrees of freedom in space, but only four driving inputs. Changing the rotation speed of any propeller will lead to the change of two attitude angles (Liu et al. 2016; Zhao and Yang 2019), so the quad-rotor helicopter is an under-actuated system. According to the structural features of the quad-rotor unmanned helicopter and the relationship between the attitude change of quadrotor and the rotation speed of each rotor, its basic motion can be divided into four basic modes:

(1) Vertical movement:

It mainly includes three flight states: vertical ascending, descending and hovering flight. When the quad-rotor fuselage is in balance state, the helicopter can rise (or fall) by increasing (or decreasing) the rotating speed of the four rotors in equal measure.

(2) Pitching movement:

Pitching movement is to keep constant rotating speeds of rotor 2 and rotor 4. Meanwhile, it 
increases (or decreases) rotor 1 and decreases (or increases) rotor 3 at the same time, but the total lifting force of four propellers remains unchanged, thus torque along the axis $y$ will be generated. The pitching movement of four rotors can be realized. Moreover, the forward and backward movement of aircraft can be realized due to the coupling effect.

(3) Rolling movement:

Rolling movement is to keep constant rotating speed of rotor 1 and rotor 3, and increase (or decrease) rotor 2 and decrease (or increase) rotor 4 at the same time, so the torque along the axis $x$ will be generated, so that the rolling movement of four rotors can be realized. Due to the coupling effect, the left and right movements of aircraft can also be realized

(4) Yawing movement:

Yawing movement is to increase (or decrease) rotor 1 and rotor 3 rotor, and decrease (or increase) rotor speed at the same time. Using the torque in opposite direction generated by the forward and reverse rotors, the torque in the overall direction of helicopter body around axis $z$ can be changed, so that yawing movement of helicopter can be achieved .

Yaw direction movement: increase (or decrease) 1 and 3 rotors, and decrease (or increase) rotor speed at the same time. Using the torque in the opposite direction generated by the forward and reverse rotors, the torque in the overall direction of the helicopter body around the shaft can be changed, so as to realize helicopter yaw movement.

All moving objects in the universe are relative. There is no movement for a single object. The position and attitude of an object in space are determined relative to another object, so the concept of reference coordinate system is generated. In order to establish the kinematic model of quad-rotor helicopter and control the attitude (Cao et al. 2016), it is necessary to introduce the definitions of two common coordinate systems.

(1) East-North-Up coordinate system $E$ :

We select a point on the ground as the reference origin, and take the horizontal plane as XOY plane. The east direction is the positive direction of $X$ axis. Rotate it 90 degrees clockwise. The north direction is the positive direction of $Y$ axis. The vertical horizontal direction is the positive direction of $Z$ axis.

(2) Aircraft-body coordinate frame $B$ :

The center of mass of quad-rotor helicopter is taken as the origin. The line between rotor 1 and rotor 3 is the axis $x$. The direction of rotor 1 is the positive direction of axis $x$. The direction of rotor 2 is the positive direction of axis $y$. The straight line passing through the origin and perpendicular to the plane $x o y$ is taken as the positive direction of axis $z$.

The attitude of rigid body describes the relative direction between two coordinate systems. The transformation of rigid body from one attitude to another can be expressed by the relative rotation transformation matrix of two coordinate systems. There are many ways to express the attitude, including quaternion, Euler angle, Euler axis/angle and direction cosine matrix. Each method has its own advantages and disadvantages. At present, the quaternion and Euler angle are commonly used. The main advantage of Euler angle representation is simple and intuitive, we only need three parameters $(\phi, \theta, \Psi)$ to represent rolling angle (Zhen et al. 2017), pitching angle and yawing angle. The disadvantage is the problem of singularity and gimbal lock, and there are a lot of trigonometric function operations in the calculation, with large calculation amount. The quaternion representation does not have these problems, and the calculation amount is small, so it can be widely applied in aerospace field and 3D animation field. The disadvantage is not intuitive enough. Each parameter 
has no actual physical significance. The attitude representation methods are introduced as follows:

(1) Euler angle representation:

Euler angle is used to describe the orientation of rigid body in three-dimensional Euclidean space. For any reference system, the orientation of a rigid body is set according to the order of rotation of three Euler angles in reference system. Therefore, the orientation of rigid body can be determined by three basic rotation matrices.

The main rotation matrices rotating around $x$-axis, $y$-axis, and $z$-axis are respectively expressed as:

$$
\begin{aligned}
C_{x}(\varphi) & =\left[\begin{array}{lll}
1 & 0 & 0 \\
0 & c_{\varphi} & s_{\varphi} \\
0 & -s_{\varphi} & c_{\varphi}
\end{array}\right] \\
C_{y}(\theta) & =\left[\begin{array}{ccc}
c_{\theta} & 0 & -s_{\theta} \\
0 & 1 & 0 \\
s_{\theta} & 0 & c_{\theta}
\end{array}\right] \\
C_{z}(\Psi) & =\left[\begin{array}{lll}
c_{\Psi} & s_{\Psi} & 0 \\
-S_{\Psi} & c_{\Psi} & 0 \\
0 & 0 & 1
\end{array}\right]
\end{aligned}
$$

The final attitude transformation matrix of rigid body is related to the order of three rotations (Wang et al. 2017; $\mathrm{Li}$ et al. 2019). In this article, the order of rotation of axis $Z-Y-X$ is adopted. The attitude transformation matrix is shown as follows:

$C_{z y x}=C_{x}(\varphi) C_{y}(\theta) C_{z}(\Psi)$

$C_{z y x}=\left[\begin{array}{lll}c_{\theta} c_{\Psi} & c_{\theta} s_{\Psi} & -s_{\theta} \\ -c_{\varphi} s_{\Psi}+s_{\varphi} s_{\theta} c_{\psi} & c_{\varphi} c_{\Psi}+s_{\varphi} s_{\theta} c_{\psi} & s_{\varphi} c_{\theta} \\ s_{\varphi} s_{\psi}+c_{\varphi} s_{\theta} c_{\psi} & -s_{\varphi} c_{\Psi}+c_{\varphi} s_{\theta} s_{\psi} & c_{\varphi c_{\theta}}\end{array}\right]$

(2) Quaternion representation:

Quaternion was a mathematical concept invented by Irish mathematician Hamilton in 1843. It shares some similarities to complex number. Quaternion is composed of three imaginary numbers and one real number. The basic expression is shown in Formula (6):

$Q=q_{0}+q_{1} i+q_{2} j+q_{3} k=q_{0}+q=\left[\begin{array}{l}q_{0} \\ q\end{array}\right]=\left[\begin{array}{l}q_{0} \\ q_{1} \\ q_{2} \\ q_{3}\end{array}\right]$

In the formula, $q_{0}, q_{1}, q_{2}$ and $q_{3}$ are the four components of $Q . q_{0}$ is the scalar part. the vector part consists of $\left[q_{1}, q_{2}, q_{3}\right]^{T}$, which are represented by symbol $q$. Corresponding to the two-dimensional space of complex number (Bernstein et al. 2018; Lin et al. 2017), if the simplest hypercomplex set such as quaternion is considered as multi-dimensional real number space, the quaternion can denote a four-dimensional space.

The quaternion that is used to represent the attitude meets the constraints in Formula (7), which is called the unit quaternion:

$\left[q_{0}^{2}+q_{1}^{2}+q_{2}^{2}+q_{3}^{2}\right]=1$ 
The multiplication symbol of quaternion is denoted by $\otimes$, the multiplication rule of quaternion can be expressed as Formula (8):

$$
Q \otimes P=\left[\begin{array}{cccc}
q_{0} & q_{3} & -q_{2} & q_{1} \\
-q_{3} & q_{0} & q_{1} & q_{2} \\
q_{2} & -q_{1} & q_{0} & q_{3} \\
-q_{1} & -q_{2} & -q_{3} & q_{0}
\end{array}\right]\left[\begin{array}{l}
p_{0} \\
p_{1} \\
p_{2} \\
p_{3}
\end{array}\right]
$$

According to the quaternion, the corresponding direction cosine matrix can be obtained by Formula (9) (Tang et al. 2019).

$$
C(Q)=\left[\begin{array}{lll}
q_{0}^{2}+q_{1}^{2}-q_{2}^{2}-q_{3}^{2} & 2\left(q_{1} q_{2}+q_{3} q_{0}\right) & 2\left(q_{1} q_{3}-q_{2} q_{0}\right) \\
2\left(q_{1} q_{2}-q_{3} q_{0}\right) & q_{0}^{2}-q_{1}^{2}+q_{2}^{2}-q_{3}^{2} & 2\left(q_{2} q_{3}+q_{1} q_{0}\right) \\
2\left(q_{1} q_{2}+q_{3} q_{0}\right) & 2\left(q_{2} q_{3}-q_{1} q_{0}\right) & q_{0}^{2}-q_{1}^{2}+q_{2}^{2}+q_{3}^{2}
\end{array}\right]
$$

Meanwhile, the following rules are defined:

$$
\left[q^{*}\right]=\left[\begin{array}{ccc}
0 & -q_{3} & q_{2} \\
q_{3} & 0 & -q_{1} \\
-q_{2} & q_{1} & 0
\end{array}\right]
$$

The air propulsive force that the four motors of the four-rotor UAV drive the propeller to rotate at high speed is the only power of aircraft. The vertical force of propeller is shown in Formula (11):

$$
T_{i}=C_{T} A_{\rho}\left(\Omega R_{r a d}\right)^{2}
$$

The reverse moment is expressed as:

$$
Q^{\prime}=C_{Q} A_{\rho}\left(\Omega R_{\text {rad }}\right)^{2} R_{\text {rad }}
$$

In general, the motor used by four-rotor helicopters is brushless dc motor, which can be approximately described by the first-order inertia link. The mathematical model of DC motor is shown in Formula (13):

$$
G(s)=\frac{0.936}{0.178 s+1}
$$

For the kinetic equation of Six-DoF aircraft, the main theoretical methods include EulerLagrange formula and Newton-Euler formula. In this paper, Newton Euler formula is used to deduce the kinetic equation.

$$
\left[\begin{array}{ll}
m I_{3 \times 3} & 0 \\
0 & I
\end{array}\right]\left[\begin{array}{l}
\& \& \\
\&
\end{array}\right]+\left[\begin{array}{l}
\omega \times m v \\
\omega \times I \omega
\end{array}\right]=\left[\begin{array}{l}
F \\
\tau
\end{array}\right]
$$

By analyzing the force of four-rotor aircraft, we can see that the force acting on the aircraft mainly comes from three sources: the lift force $F_{T}$ generated by rotor wing, the gravity force $F_{G}$ of aircraft, and the air resistance force $F_{D}$ in flight. The resultant external force in total inertial coordinate system can be expressed as:

$$
F=C_{b}^{e} F_{T}-F_{G}-F_{D}
$$

In the formula, $C_{b}^{e}$ denotes the directional cosine matrix from the airframe coordinate system to the inertial coordinate system. Respectively, the air resistance, the lift force of rotor and the gravity vector are:

$F_{D}=K_{d} V$ 
$F_{T}=\sum_{i=1}^{4} T_{i}$

$F_{G}=[0,0, m g]^{T}$

The inertia tensor of a rigid body in space can be expressed as:

$I=\left[\begin{array}{l}I_{x x}, I_{x y}, I_{x z} \\ I_{y x}, I_{y y}, I_{y z} \\ I_{z x}, I_{z y}, I_{z z}\end{array}\right]$

In order to simplify the system model, we can assume that the mass center of the four-rotor helicopter is in the center of airframe, and its structure has good symmetry. If we only consider the rotational inertia of main shaft, the inertial product in Formula (19) $I_{x y}=I_{y x}=I_{z x}$, and the inertia tensor matrix is a diagonal matrix. Combined with the optimal iterative learning algorithm based on the discrete equation of quadratic performance function in time domain, the estimated model error is compensated based on disturbance, and then UAV trajectory big data tracking is completed.

2.2 Big data tracking and automatic measurement technology of UAV trajectory based on MEMS sensor

Based on the analysis of Section 2.1, the error function is established. Firstly, the acceleration of gravity and the intensity of magnetic field in the ground reference coordinate system must be converted to the aircraft-body coordinate frame. This conversion is based on the rotation matrix. Then, the acceleration value and magnetic field intensity measured by accelerometers and magnetometers in coordinate system are subtracted, so that the error function can be obtained.

Acceleration error function:

$\left.\left.f_{g}\left(\begin{array}{c}b \\ e\end{array}\right), a^{b}\right)={ }_{e}^{b} q\right) \otimes g^{e} \otimes_{e}^{b} q-a^{b}$

In formula, $\begin{array}{ll}b \\ e\end{array} q$ denotes the quaternion from the ground reference coordinate system $E$ to the airframe coordinate system $B ; g^{e}$ is the acceleration of gravity in the ground reference coordinate system, and it should be standardized: $g^{e}=[0,0,0,1] ; \quad a^{b}$ is the acceleration value measured by the accelerometer in the airframe coordinate system, which is expressed as:

$a^{b}=\left[0, a_{x}, a_{y}, a_{z}\right]$

The acceleration error function is:

$\left.f_{g}\left(\begin{array}{c}b \\ e\end{array}\right), a^{b}\right)=\left[\begin{array}{l}2\left(q_{1} q_{3}-q_{0} q_{2}\right)-a_{x} \\ 2\left(q_{0} q_{1}+q_{2} q_{3}\right)-a_{y} \\ 2\left(1 / 2-q_{1}^{2}-q_{2}^{2}\right)-a_{z}\end{array}\right]$

According to the derivation of Formula (23), its Jacobian matrix can be obtained. 
$J_{e}\left(\begin{array}{l}b \\ a\end{array} q^{e}\right)=\frac{d f_{k}\left(\left(\begin{array}{l}b \\ a\end{array} q^{e}\right)\right)}{d_{p}^{e}}=\left[\begin{array}{l}-2 q_{2}, 2 q_{3}, 2 q_{0}, 2 q_{1} \\ 2 q_{1}, 2 q_{0}, 2 q_{3}, 2 q_{2} \\ 0,-4 q_{1},-4 q_{2}, 0\end{array}\right]$

Error function of magnetometer:

$f_{m}\left({ }_{e}^{b} q+b^{e}, m^{e}\right)={ }_{e}^{b} q \otimes m_{p}^{p}$

Combined with the above analysis, a differential equation is established by the measured value of gyroscope, and a set of quaternions is obtained by solving this equation. Then, the error functions of accelerometer and magnetometer are established. The gradient descent method is used to solve this error function, and a set of quaternions can be obtained. According to related theories, no matter MEMS gyroscope, accelerometer or magnetometer, there are some errors in measured values. Therefore, it is necessary to fuse these quaternions to get more accurate quaternions, so as to get the measurement results. The attitude fusion algorithm based on gradient is:

${ }_{e}^{b} q_{e s t}=\gamma_{e}^{b} q \Delta(k)+(1-\gamma)_{e}^{b} q_{\omega}$

In the above formula: ${ }_{e}^{b} q_{\omega}$ is the attitude quaternion solved by gyroscope quaternion differential equation; $\gamma_{e}^{b} q \Delta(k)$ is the attitude quaternion solved by gradient descent method; $(1-\gamma)$ and $\gamma$ are the weights of two attitude quaternions.

$\mu / T_{k}$ is set as the convergence rate of $\gamma_{e}^{b} q \Delta(k)$, and it also represents the measurement error of gyroscope. Based on the convergence rate and divergence angle of optimal attitude solution, Formula 26 can be obtained:

$(1-\gamma) \beta=\gamma \frac{\mu(k)}{T_{s}}$

Then,

$\gamma=\frac{\beta}{\frac{\mu(k)}{T_{s}}+\beta}$

In order to ensure the accuracy of gradient descent method, we must ensure that the convergence speed of gradient descent method is faster than the moving speed of object. Once the motion speed of object is greater than the convergence speed of gradient descent method, the gradient descent method will lose the tracking ability. In order to ensure that the convergence rate of gradient descent method is sufficiently large, the step length must be large enough. If the step length is sufficiently large, $\alpha$ must be sufficiently large. In this way, if the object moves at high speed, the algorithm can track it. But $\alpha$ can't be too large. If $\alpha$ is too large, it will lead to poor static performance of system. When $\alpha$ is large, $\mu(k)$ is large in the relevant formula. Therefore, the previous quaternion can be ignored, and it can be simplified as:

${ }_{e}^{b} q_{\nabla}(k)=-\mu(k) \frac{\nabla f}{\|\nabla f\|}$ 
Similarly, Formula (27) can be simplified as:

$\gamma=\frac{\beta T_{s}}{\mu(k)}$

After the above formulas are combined, the final attitude fusion formula of gradient descent method can be obtained:

${ }_{e}^{b} q_{e s t}(k)={ }_{e}^{b} q_{e s t}(k-1)+{ }_{e}^{b} q_{\omega}(k)-\beta \frac{\nabla f}{\|\nabla f\|} T_{s}$

The direction of magnetic field in ground reference coordinate system is constant. The intensity of magnetic field changes with the change of UAV position in flight. Meanwhile, the strength of magnetic field in ground reference coordinate system is also changing. It is very easy to be disturbed by the environment. In the process of practical application, it is necessary to know the magnetic field intensity in ground reference coordinate system. It is unnecessary to measure directly the magnetic field intensity, but to use the magnetometer to measure the magnetic field intensity. Firstly, it is converted to the ground reference coordinate system, and the magnetic field intensity in ground reference coordinate system is obtained, and then it is converted to the airframe coordinate system for error correction.

If the magnetic field intensity measured by the magnetometer in body coordinate system is $m^{b}(k)$, and the magnetic field intensity in ground reference coordinate system after rotation is $h^{e}$, then the following relationship is established:

$h^{e}=\left[0, h_{x}, h_{y}, h_{z}\right]$

For the magnetic field in ground reference coordinate system, if its intensity is $b^{e}(k)$, thus:

$$
b^{e}(k)=\left[0, \sqrt{h_{x}^{2}+h_{z}^{2}}, 0, h_{z}\right]
$$

The gradient descent method is used to process the acceleration data and magnetometer data, so as to obtain the optimal quaternion. And then, the two quaternions are fused by MEMS sensor. Finally, the measurement results can be obtained.

$$
\left\{\begin{array}{l}
x(t)=f(t)+u(t)+t \\
y(t)=g(t)+u(t)+t
\end{array}\right.
$$

\section{Simulation experiment}

In order to verify the comprehensive effectiveness of the proposed method, the simulation experiment was carried out. Experimental environment: SGHz P4CPU,slZM, 128M memory, GeForee4Ti4200withAGPSX, 1024x768 screen resolution, 17x17 terrain block, 256x256 texture block.

(1) big data tracking errorofUAV trajectory/ (\%):

Next, the simulation platform of four-rotor unmanned helicopter was constructed by MATLAB software, and various measuring data was verified. The comparison results of big data tracking errors of three methods are given in the following tables:

Table 1. Big data tracking error of UAV trajectory of the proposed method 


\begin{tabular}{cc}
\hline Number of iterations /(times) & Big data tracking error of UAV trajectory /(\%) \\
\hline 1 & 0.0012 \\
2 & 0.0010 \\
3 & 0.0008 \\
4 & 0.0007 \\
5 & 0.0006 \\
6 & 0.0005 \\
7 & 0.0004 \\
8 & 0.0002 \\
9 & 0.00 \\
10 & 0.00 \\
\hline
\end{tabular}

Table 2. Big data tracking error of UAV trajectory of reference (Dai et al. 2019)

\begin{tabular}{cc}
\hline Number of iterations /(times) & Big data tracking error of UAV trajectory /(\%) \\
\hline 1 & 0.012 \\
2 & 0.014 \\
3 & 0.015 \\
4 & 0.018 \\
5 & 0.021 \\
6 & 0.024 \\
7 & 0.027 \\
8 & 0.030 \\
9 & 0.033 \\
10 & 0.036 \\
\hline
\end{tabular}

Table 3. Big data tracking error of UAV trajectory of reference (Zhou et al. 2019)

\begin{tabular}{cc}
\hline Number of iterations /(times) & Big data tracking error of UAV trajectory /(\%) \\
\hline 1 & 0.014 \\
2 & 0.018 \\
3 & 0.023 \\
4 & 0.027 \\
5 & 0.031 \\
6 & 0.036 \\
7 & 0.040 \\
8 & 0.044 \\
9 & 0.047 \\
10 & 0.050 \\
\hline
\end{tabular}

After analyzing above experimental data, we can see that the big data tracking error of UAV trajectory of the proposed method is the lowest. The error of the method in Reference [5] is the second. The error of the method in Reference [6] is the highest.

(2) Measurement error / (\%):

In order to further verify the effectiveness of the proposed method, it is necessary to compare the measurement errors of the three methods. The comparison results are shown in Fig. 1: 


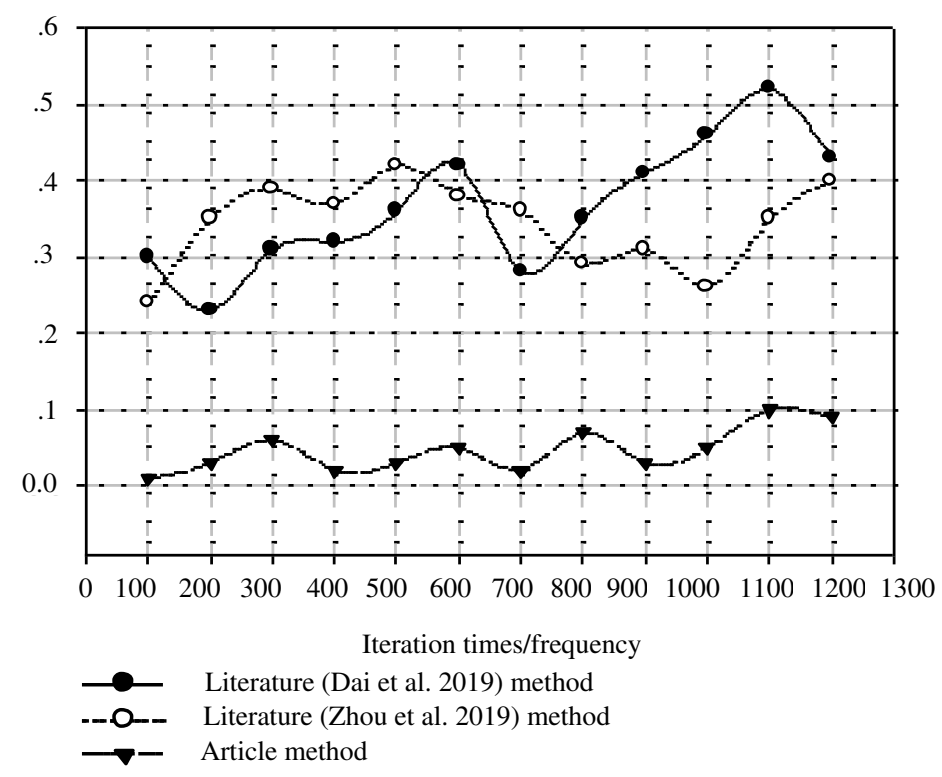

Fig.1 Comparison result of measurement errors of different methods

From Fig. 1, we can see that the measurement errors of various methods are also changing with the iteration times. When the number of iterations was 100 , the measurement error of the method in Reference (Dai et al. 2019) is 0.3, the measurement error of the method in Reference (Zhou et al. 2019 ) is 0.24 , and the measurement error of the proposed method is 0.01 . When the number of iterations is 1200 , the measurement error of the method in Reference (Dai et al. 2019) is 0.43, the measurement error of the method in Reference (Zhou et al. 2019) is 0.40, and the measurement error of the proposed method is 0.09 . The measurement error of the proposed method is always low, so the proposed method has high measurement accuracy.

(3) Running time / ( $\mathrm{min})$ :

Based on above experimental conditions, the running time of the three methods is compared. The comparison results are shown in Table 4:

Table 4. Change of running time of different methods

\begin{tabular}{cccc}
\hline Number of samples & $\begin{array}{c}\text { The propose } \\
\text { method }\end{array}$ & $\begin{array}{c}\text { Running time/(min) } \\
\text { Method in Reference } \\
\text { (Dai et al. 2019) }\end{array}$ & $\begin{array}{c}\text { Method in Reference } \\
\text { (Zhou et al. 2019) }\end{array}$ \\
\hline 100 & 1.23 & 1.45 & 1.65 \\
200 & 1.24 & 1.48 & 1.72 \\
300 & 1.27 & 1.52 & 1.84 \\
400 & 1.30 & 1.57 & 1.92 \\
500 & 1.32 & 1.64 & 2.03 \\
600 & 1.35 & 1.70 & 2.10 \\
700 & 1.37 & 1.75 & 2.21 \\
800 & 1.40 & 1.82 & 2.32 \\
900 & 1.42 & 1.90 & 2.42 \\
1000 & 1.45 & 1.95 & 2.55 \\
1100 & 1.48 & 1.99 & 2.64 \\
1200 & 1.51 & 2.03 & 2.75
\end{tabular}


According to analysis of experimental data, we can see that the running time of measurement method is changing with the increasing number of samples. The running time of the proposed method is the lowest in three methods, so that the high efficiency of the proposed method can be proved.

\section{Conclusions}

Due to flexibility, good motility, high safety, simple structure, miniaturization and other characteristics, the four-rotor helicopter is more and more favored by researchers. In recent years, with the improvement of technology of aerial robot and the successful application in some fields, the development of quadrotor UAV will be more and more rapid. Based on previous researches, this article focuses on four-rotor helicopter trajectory tracking measurement, multi-sensor data fusion method and sensor data processing method, mainly including the following aspects:

(1) According to the dynamic model of quadrotor, the repetitive disturbance and unmodeled error of system are estimated in the neighborhood space by the optimal estimation characteristics of Kalman filter. The optimal iterative learning algorithm based on quadratic performance function in time domain is used to compensate the estimated model error, and Matlab software is used to evaluate the learning algorithm. The tracking results of four-rotor unmanned helicopter under various trajectories are given by simulation, so that the effectiveness of the proposed algorithm can be proved.

(2) After finishing the theoretical simulation, an experimental platform for the flight control system of four-rotor unmanned helicopter is built. According to the measurement characteristic of sensor, the sensor is calibrated to obtain the final experimental result. Simulation results fully prove the effectiveness and superiority of the proposed method.

The design of flight control system of four-rotor helicopter is a long-term task. Due to the limitation of personal time and energy, and the experimental conditions, there are many expansions works to be improved.

(1) it is necessary to improve and perfect the hardware platform of flight control system. More accurate sensors and better filtering algorithm should be adopted. Meanwhile, the computer vision should be added to provide more accurate position and attitude for the aircraft, thus making the aircraft more stable and intelligent.

(2) Furthermore, the path planning of four-rotor helicopter can be added, and the iterative learning method can be combined with the proposed method to improve the robustness of system.

\section{Ethical approval}

This article does not contain any studies with human participants performed by any of the authors.

\section{Conflict of interest}

The authors declare no conflict of interest.

\section{References}


Bernstein J, Bancu M, Cook E, Duwel A, Elliott R, Gauthier D, Golmon S, Leblanc J, TomainoIannucci M, Ung J, Weinberg M (2018) Resonant acoustic MEMS wake-up switch. J Micro electromechanical Syst (99)1-10.

Cao RC, Chen J, Wang YT (2016) A hybrid tracking and positioning algorithm for mobile terminals. J Taiyuan Univ Technolog 47(4):506-512.

Dai SX, Zhang YJ, Peng DS, Wu F, Ma WX, Xu Y (2019) Target tracking and localization algorithm for UAV based on RPCCA. Transducer Microsyst Technolog 38(04):138-140+144.

He DG, Wang GH, Sun DX (2016) Distributed radar network target tracking technology under distributed interference. Mod Def Technol 44(4):81-88.

Jiang T, Jie L, Huang K (2015) Longitudinal parameter identification of a small unmanned aerial vehicle based on modified particle swarm optimization. Chinese J Aeron 28(003):865-873.

Lei X, Lang B, Du Y et al (2011) A small unmanned polar research aerial vehicle based on the composite control method. Mechatron 21(5):821-830.

Li JL, Yin K, Chu CX (2019) Review of video target tracking technology. J Yanshan Univ 43(3):251-262.

Lin LP, Huang TQ, Lin J (2017) Target tracking algorithm combining foreground discrimination and circular search. Comput Appl 37(11):3128-3133.

Liu WJ, Dong SH, Qu HC (2016) Spatio-temporal contextual anti-occlusion visual tracking. J Image Graph 21(8):1057-1067.

Nie GH (2019)Analysis of landing trajectory tracking method for fully autonomous dual-fluid fourrotor UAV. J China Acad. Electron Inf Technolo 14(2):134-138.

Niu TF, Zhou YS, Qu C (2019)Research on acceleration extraction technology based on MEMS sensor. Electron Meas Technol 42(07):130-134.

Pang YF, Li CF, Niu PF (2016) Aircraft measurement and control memory tracking algorithm based on theoretical ballistic speed matching. Telecommun Technolog 56(4):443-447.

Peng Y, Shi SH, Guo K, Liu DT (2019) Fault simulation and data generation of UAV flight control system. Chin J Sci Instrum 40(07):13-21.

Tang Y, Ten CW, Schneider K (2019) Inference of tampered smart meters with validations from feeder-level power injections. IEEE Pes Isgt Asia 5:1-6.

Wang SL, Bi DP, Ruan HL (2017) Adaptive data correlation tracking algorithm for cognitive radar waveforms. J Astronaut 38(12):1331-1338.

Zhai R, Zhou Z, Zhang W et al (2014) Control and navigation system for a fixed-wing unmanned aerial vehicle. AIP Adv 4(3):36.

Zhao YF, Yang MZ (2019) Unmanned aerial vehicle trajectory prediction based on operating state recognition. Sci Tech Eng 19(23):304-309.

Zhen ZY, Tao G, Jiang J (2017) Adaptive tracking control of UAV's automatic landing on the net. J Harbin Eng Univ 38(12):1922-1927.

Zhou SQ, Hua YZ, Dong XW, Li QD, Ren Z (2019) Air-ground time varying formation tracking control for heterogeneous UAV-UGV swarm system. Aero Weaponry (4):54-59.

Zuo L, Sun HK, He YG, Yin BQ, Hu XM (2019) Reliability analysis of MEMS sensor without failure data. J Electron Meas Instrum 33(06):69-75. 


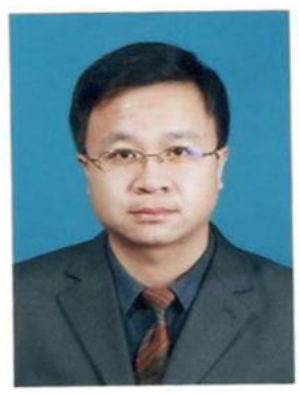

Jun Xing male 1972.9. He received the B.Sc. and M.Sc. degrees in Computer Technology and Application from Northeastern University in 1996and 1999. He received the Ph.D., degrees in Computer Technology and Application from Dalian University of Technology in 2008, respectively. $\mathrm{He}$ is now an Associate Professor with the School of Information Science and Engineering, Dalian Polytechnic University, China. His research interests include research and application of big data algorithm.

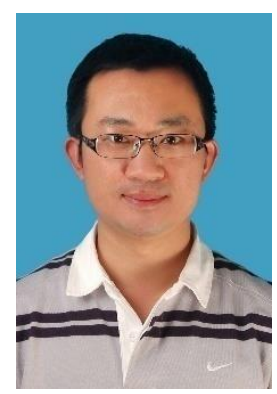

Xinzhe Wang, Male 1982.3. He received the Ph.D degree from the School of Control Science and Engineering, Dalian University of Technology, Dalian, China, in 2012. He is currently a Lecturer in the School of Information Science and Engineering, Dalian Polytechnic University, Dalian, China. His research interests include big data, data driven modeling and deep learning neural networs.

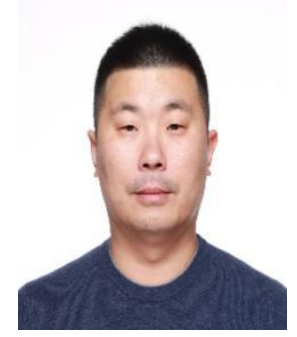

Jie Dong, Male 1981.1. He received the Ph.D degree from the School of Control Science and Engineering, Dalian University of Technology, Dalian, China, in 2009. He is currently aAssociate Professor in the School of Information Science and Engineering, Dalian Polytechnic University, Dalian, China. His research interests include big data modeling and data mining. 
Figures

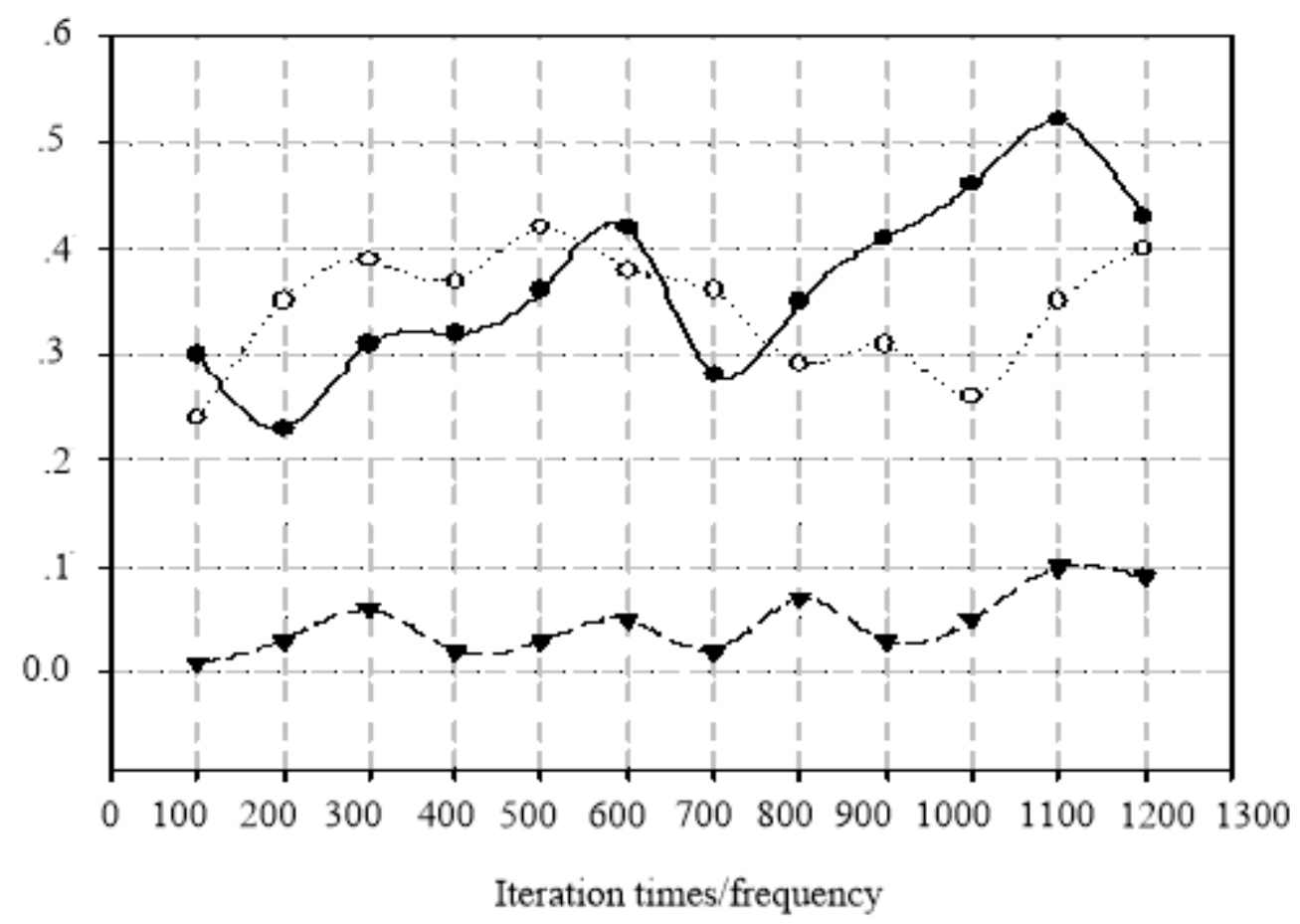

$\longrightarrow$ Literature (Dai et al. 2019) method

... O... Literature (Zhou et al. 2019) method

- $\mathbf{-}$ Article method

Figure 1

Comparison result of measurement errors of different methods 